\title{
Health-Related Quality of Life in Morphea
}

\author{
N.K. Klimas ${ }^{1}$, A.D. Shedd ${ }^{2}$, I.H. Bernstein ${ }^{3}$, and H. Jacobe ${ }^{1}$ \\ ${ }^{1}$ Department of Dermatology, University of Texas Southwestern Medical School, Dallas, Texas \\ 2ProPath Associates, Dallas, Texas \\ ${ }^{3}$ Department of Clinical Science, University of Texas Southwestern Medical School, Dallas, Texas
}

\section{Abstract}

Background-Little is known about the health-related quality of life (HRQOL) of patients with morphea, and previous studies have yielded conflicting results.

Objectives-To determine the impact of morphea on HRQOL and clinical and demographic correlates of HRQOL in adults.

Methods-Cross sectional survey $(n=73)$ of Morphea in Adults and Children (MAC) cohort.

Results-Morphea impairs HRQOL in adults. Patients were most impaired by emotional wellbeing and concerns that the disease will progress to their internal organs. Patients with morphea had worse skin-specific HRQOL than those with non-melanoma skin cancer, vitiligo, and alopecia (lowest $P<.0001$ ). Study subjects had significantly worse global HRQOL scores than the general U.S. population for all subscales (all $P \leq 004)$ with the exception of bodily pain. Comorbidity $(r=$. $35-.51, P \leq .0029-.0001)$ and symptoms of pruritus $(r=.38-.64, P \leq 001-.0001)$ and pain $(r=.46-$. $74, P<.0001)$ were associated with impairment in multiple domains of skin-specific and global HRQOL. Physician-based measures of disease severity correlated with patient-reported HRQOL.

Conclusion-Patients with morphea have negative impact on HRQOL particularly if symptoms (pruritus and pain) or concerns regarding internal manifestations are present. Providers should be aware of this when evaluating and treating patients.

\section{Key Indexing Terms}

Scleroderma; Localized; Severity of Illness Index; Quality of Life; Adult; Health Surveys; CrossSectional Studies; morphea; Skindex-29; SF-36; itch

\section{INTRODUCTION}

Morphea, also known as localized scleroderma, is an idiopathic inflammatory disorder that produces sclerosis of the skin and subcutaneous tissues. Recent studies have examined physician based outcome measures in morphea including clinical and radiology based

Corresponding Author: Heidi Jacobe, MD, MSCS, Associate Professor, Department of Dermatology, UT Southwestern Medical Center, 5323 Harry Hines Blvd., Dallas, TX 75390-9069, Ph: 214-648-3324, Fx: 214-648-5559, heidi.jacobe@ utsouthwestern.edu. Conflict of Interest:

The authors report no conflicts of interest. 
outcomes. Few have examined the impact of morphea on health-related quality of life (HRQOL), particularly in adults.

It is well-known that cutaneous disease impacts HRQOL. ${ }^{1-3}$ Further, the importance of patient based outcomes has been underscored in numerous publications. ${ }^{4}$ Yet HRQOL in morphea is poorly described, and existing studies largely focus on children. ${ }^{5-7}$ These pediatric studies indicate that morphea has modest effect on life quality, while studies in adults generally report greater negative impact on life quality and emotional distress. 6,8 Conclusions from these studies are limited by incomplete information regarding patient demographics and clinical features or by use of unidimensional measures. ${ }^{6,8-11}$ No studies to date have examined the impact of morphea on overall HRQOL in adults, thus comparison of impact of morphea with that of other medical conditions is limited. Further, correlation between newly validated clinical scoring systems with patient perceived disease impact is poorly described.

The objective of this study was to determine the impact of morphea on HRQOL in adults as measured by multidimensional HRQOL scales (Skindex-29+3 and SF-36) and ascertain how well these measures correlated with physician based outcomes. We also determined demographic and clinical features correlated with HRQOL.

\section{MATERIALS AND METHODS}

\section{Participants}

The institutional review board-approved Morphea in Adults and Children (MAC) cohort contained 322 adults ( $\geq 18$ years old at enrollment) and children ( $\leq 17$ years old at enrollment) as of March 2012. Criteria for inclusion in this study included: eligibility for enrollment in MAC cohort (the details of eligibility have been reported previously), 8,12 age $\geq 18$ years at time of enrollment, presence of sufficient information for analysis including baseline Skindex- 29+3 and SF-36 HRQOL surveys, and English language/literacy skills. Of the 322 of patients in the cohort, 249 were excluded due to the following: age < 18 years $(n=79)$, English language/literacy skills $(n=4)$, and insufficient data on variables of interest $(n=166$, the Skindex-29+3 and SF-36 were not administered at the outset of the cohort).

The MAC cohort was designed to prospectively capture prevalent and incident cases of morphea. Patients are recruited from within the UT Southwestern Medical Center system and through regional referrals from private practitioners.

After patients signed consent, all data were abstracted using a comprehensive clinical report form designed prior to the study, including demographic, clinical, medical and family history, and HRQOL questionnaires. All patient reported histories were also confirmed by review of medical records. At the time of enrollment, all patients were evaluated by one examiner with expertise in morphea (H.J.), who assigned subtype and clinical scores (modified Rodnan skin score and LoSCAT). ${ }^{13,14}$ Subtype classifications used herein are those proposed by Zulian and Laxer). ${ }^{15}$ 


\section{Variables of Interest}

Skindex-29+3-Skin-specific HRQOL was evaluated with the Skindex-29. ${ }^{16}$ The Skindex-29 assesses three subscales: emotions, symptoms, and functioning in the month preceding administration. A fourth subscale was added to characterize morphea-specific concerns: (1) activity limitation as a result of disease, (2) concern for involvement of internal organs and (3) feelings of isolation. Morphea specific subscale items were initiated by the investigators based on concerns commonly expressed by patients. Each subscale score ranges from 0 to 100 ; higher scores indicate poorer HRQOL.

DLQI-Skin-specific HRQOL was also measured with the Dermatology Life Quality Index (DLQI) ${ }^{17}$ This questionnaire evaluates the impact of skin disease on HRQOL over the week prior to administration. Total score ranges from 0 to 30 and higher scores indicate poorer HRQOL.

SF-36-HRQOL as it pertains to general health was measured via the SF-36. ${ }^{18}$ This $36-$ item questionnaire evaluates eight health domains: physical functioning (PF), role-physical (RP), bodily pain (BP), general health (GH), vitality (VT), social functioning (SF), roleemotional (RE), and mental health (MH). The eight scales of the SF-36 are incorporated in two summary measures, the Physical Component Summary (PCS), and the Mental Component Summary (MCS). SF-36 scores range from 0 to 100; lower scores indicate poorer HRQOL.

SCQ-The burden of patients' comorbidities was measured with the Self-Administered Comorbidity Questionnaire (SCQ). ${ }^{19,20}$ Scores range from 0 to 45 , with higher scores indicating maximal comorbidity. ${ }^{20}$ The SCQ assesses the presence (and limitations imposed by) prevalent conditions including heart disease, hypertension, and back pain.

\section{Pruritus and Pain}

Physical symptoms of pruritus and pain were assessed on a visual analog scale (VAS) of 1-10, with 10 representing greatest severity. Visual analog scales for patient reported symptoms were utilized because they have extensive validation and history of use in skin disorders, including prior studies with morphea in the form of the Impact of Chronic Skin Disease on Daily Life (ISDL). ${ }^{9,10,21-23}$

\section{Physician Based Measures}

Patients were scored by the same investigator (H.J.) using the modified Rodnan Skin Score $(\mathrm{mRSS})^{13}$ and the newly validated Localized Scleroderma Cutaneous Assessment Tool (LoSCAT). ${ }^{14}$ Because the LoSCAT was not validated at the inception of the cohort, patients enrolled before 2008 were only scored with the mRSS. After 2008, MAC patients were assessed with both the mRSS (for continuity with initial assessments) and the LoSCAT. The LoSCAT contains measures of activity (Localized Scleroderma Skin Severity Index [LoSSI] and Physician Global Assessment-Activity [PGA-A]) and damage (Localized Scleroderma Skin Damage Index [LoSDI] and Physician Global Assessment-Damage [PGA-D]). Higher scores on all physician-based measures indicate greater disease severity. ${ }^{14,24}$ 
Cosmetically sensitive areas were identified as lesions on the face and neck. Functional limitation was defined as having at least one of the following: (1) limited joint mobility (limited range of motion secondary to skin and subcutaneous tissue involvement, but not due to arthritis or other mechanical issues), (2) limb-length discrepancy or (3) contracture.

\section{Socioeconomic Variables}

Through use of the subjects' residential postal zip codes and 2007-2011 U.S. census data (http://factfinder2.census.gov), annual household income was obtained for each zip code tabulation area as an aggregation of census tracts using previously published methods. ${ }^{25,26}$ As census data is not normally distributed, median values were used and classified as $\leq$ $\$ 24,999 /$ year, $\$ 25,000-\$ 49,999 /$ year, $\$ 50,000-\$ 99,999 /$ year, and $\$ \$ 100,000 /$ year. As an additional marker of socioeconomic status we categorized participants' insurance as public, private, or uninsured. ${ }^{27,28}$

\section{Statistical Analysis}

Overall HRQOL in morphea was evaluated by summary statistics of the Skindex-29+3, DLQI, and SF-36 and assessing correlations among subscales. HRQOL in morphea was compared with historical HRQOL in other dermatologic diseases by comparing means of the three Skindex-29 subscales. ${ }^{22,29}$ HRQOL in morphea was compared with that of other medical conditions, and with the general U.S. population using norm-based scores of the SF-36. ${ }^{30}$ Data for comparison was obtained from previous studies evaluating HRQOL. ${ }^{31-33}$ Means were compared using a two-tailed, two-sample $t$ test.

We also evaluated the relationship between HRQOL (defined as a dependent variable) and independent variables (including demographic and clinical) of interest. Associations with HRQOL were assessed using analysis of variance (ANOVA); two-tailed, two-sample $t$ tests; and Pearson correlations (to minimize experiment-wise error rate, comparisons between morphea and other diseases were evaluated with statistical significance designated as $P<$. 01). Calculations were completed using SAS 9.3 (SAS Institute, Cary, NC).

\section{RESULTS}

\section{Patient Characteristics}

Seventy-three patients out of 322 met inclusion criteria, the majority were female (85\%), white (71\%), and with the generalized morphea subtype (59\%). Details of the demographic and clinical features of subjects are available in Table 1. The majority of our subjects had private insurance and moderate to high income. Although there were differences in the mean HRQOL scores based on demographics (ethnicity and income), they did not reach statistical significance. Patients were treated with topical/intralesional therapies (including calcipotriene, imiquimod, topical tacrolimus, intralesional and topical corticosteroids), systemic immunosuppressives (including methotrexate, systemic and intramuscular corticosteroids) and/or phototherapy, or were not undergoing active treatment. Patients on multiple therapies were counted more than once (Figure 1). 


\section{Skin-Specific HRQOL}

Skindex 29+3-Among the Skindex-29+3 subscales, highest mean scores occurred in the emotions and morphea-specific domains, with mean + SD scores of $41+26$ and $37+29$, respectively (Figure $2 \mathrm{~B}$ ). Within the emotions domain, patients were most concerned that their skin may get worse $(63+34)$ or that their condition may be serious $(55+33)$ (Fig. 1B). In the morphea-specific domain, patients were most concerned that the condition would affect their internal organs (49+35) (Figure 1C). In the symptoms domain, patients were most affected by itch $(47+31)$; patients noted their social life was affected within the functioning domain $(32+32)$.

Skindex-29 scores in morphea were compared to patients with other dermatologic conditions and those without skin disease (Table 2). Ethnicity ${ }^{29}$ and gender ${ }^{22,29}$ distributions of our cohort are similar to historic reference populations. Patients with morphea had greatest impairment in the emotions domain, with Skindex-29 mean+SD scores $(41+26)$ similar to those with acne vulgaris $(41+25)$, eczema $(40.8+27)$ and psoriasis $(39+27)$. HRQOL in morphea was significantly poorer across all subscales of the Skindex-29 than those without dermatologic disease $(P<.0001)$.

DLQI-Thirty four patients (47\%) experienced moderate or greater impact on HRQOL as defined by a DLQI $>5 .{ }^{17}$ The details of studies examining DLQI in this cohort were previously published. ${ }^{8}$

\section{Overall HRQOL}

SF-36 scores in morphea were compared with the general U.S. population, healthy individuals, and those with 9 other medical conditions (Table 3). With respect PCS, patients with morphea were similar to those with pain (sciatica) and depression and significantly worse than the U.S. healthy population. ${ }^{32}$ Patients with morphea had greatest impairment in the mental health domain. With regard to MCS, scores of patients with morphea were similar to those with systemic lupus erythematosus, and significantly poorer than the general and healthy U.S. population. ${ }^{31,32}$

\section{Multivariate Analysis (Table 4)}

Patient-based measures of HRQOL (Skindex-29+3, DLQI, SF-36), were highly correlated with one another $(P<.0001)$. Correlation among the emotions, symptoms, functioning, and morphea-specific Skindex 29+3 subscales was of relevant magnitude of effect, as high scores in one subscale were associated with high scores in others $(\mathrm{r}=44770-6995, P<.0001)$.

\section{Clinical Variables Correlated with HRQOL (Table 4)}

Patient-reported comorbidity as identified by the SCQ correlated with impaired HRQOL across 2 Skindex- $29+3$ subscales (with the exception of emotions), the DLQI, and both summary measures of the SF-36. Lesion pain was associated with impairment in the SF-36 PCS, DLQI and all Skindex-29+3 subscales with the exception of emotions. Presence of itch was associated with worse Skindex 29+3 symptoms and morphea-specific Skindex-29+3 scores. Morphea specific concerns, including activity limitation, were closely linked with 
the Skindex functioning subscales and SF-36 PCS (r= 6995 and .5706 , respectively, $P<$. 0001).

Increased severity of active disease (LoSSI component) correlated with worse HRQOL. The LoSSI was associated with worse symptoms and morphea-specific Skindex-29+3 subscores and worse SF-36 PCS scores. There was little correlation with HRQOL and the damage domain of the LoSCAT (LoSDI) with exception of the SF-36 PCS. Physician-assessed functional limitation was associated with worse morphea-specific Skindex-29+3 scores and SF-36 PCS scores.

Patients with generalized disease had significantly poorer HRQOL than those with the plaque subtype in the Skindex 29+3 morphea-specific domain (Fig 2D). There were no significant differences in HRQOL between patients receiving treatment and those that were not.

\section{DISCUSSION}

In this prospective cross sectional study of adults enrolled in the MAC cohort we determined the impact of morphea on HRQOL and associated demographic and clinical features. Our results demonstrated that morphea had negative impact on HRQOL similar to disorders such as eczema and rheumatoid arthritis, especially in the domain of emotions and mental health. The presence of comorbid conditions, increased morphea activity, and symptoms were correlated with impaired HRQOL across all measures.

We confirmed prior observations that symptoms associated with morphea, particularly pain and itch were strongly associated with poor HRQOL.${ }^{8-10}$ In fact, pain and itch were stronger correlates of HRQOL than the location of lesions in cosmetically or functionally sensitive sites. This likely reflects the notion that physical symptoms are known pervasive, chronic stressors linked with anxiety, depression, and impairment of activities of daily living. ${ }^{34-36}$ Similar to prior observations in our cohort, itch was significantly correlated with lesion activity (as measured by the LoSSI component of the LoSCAT), suggesting that pruritus might be a marker of active disease. Similar to studies in other disorders, the presence of greater comorbidity was strongly associated with greater impairment, though common conditions (e.g., hypertension) were unrelated to morphea. This implies patients have difficulty discerning the impact of morphea in the context of multiple health concerns. This may also account for the lesser impact of morphea on HRQOL in children versus adults. ${ }^{37}$

We also found that increased disease activity as measured by the LOSSI component of the LoSCAT is linked with poorer HRQOL in morphea. Our results are similar to those of Szramka-Pawlak et al, who found a correlation between the activity domain of the LOSSI and skin-specific HRQOL in their population as measured by the Skindex-29. ${ }^{11}$ In contrast to previous observations in our cohort in which DLQI scores were correlated with the presence of damage as measured by the LoSDI, the results of the present study suggest that the LOSSI (activity) is more closely linked with HRQOL than the LoSDI (damage) ${ }^{8}$ although functional impairment (which is not a component of the LoSDI) did have negative impact on HRQOL and may be considered damage. This emphasizes the importance of 
addressing active lesions in clinical practice and implies the LoSCAT activity measures elements of morphea significant to patients but the LoSDI may not.

The finding that socioeconomic status was not associated with impact on HRQOL was unexpected, as these factors are known to strongly affect HRQOL. ${ }^{38}$ However, despite our efforts to recruit a diverse patient population, our sample was predominately Caucasian and relatively affluent, which may reflect the referral center setting of our study or even the relatively high socioeconomic status of the patient population in Dermatology as a whole. ${ }^{39}$ This might have limited our ability to determine the effect of these variables.

We are not aware of any prior studies that examined the effect of morphea on global HRQOL in adults. This has prevented comparison between morphea and other medical conditions. We found that morphea impacts overall HRQOL, particularly its mental domains, to an extent comparable to a number of conditions with frequent internal manifestations such as back pain and rheumatoid arthritis. Similarly, comparison of Skindex-29 scores reveals morphea has impact resembling other chronic skin conditions such as vitiligo and alopecia. These findings confirm that morphea has serious implications in the view of affected patients.

A notable finding from our study is that participants had a high level of concern that morphea may affect their internal organs. This may reflect the ability of patients to independently research morphea, at which point they likely encounter sources referring to morphea interchangeably with localized scleroderma, systemic sclerosis, and scleroderma which produces confusion with regard to diagnosis and prognosis. This highlights the need for providers to educate patients appropriately, particularly with respect to differences between morphea and scleroderma.

The present study includes a number of limitations. Sample size limits conclusions in subgroups of patients. Generalized morphea was more prevalent in our cohort than the morphea population as a whole, likely because our study was conducted at a referral center. Thus HRQOL in the MAC cohort may be poorer than that of patients with morphea overall. Also, the cross sectional nature of the study precludes conclusions regarding the effect of time and treatment on HRQOL.

This study demonstrates that morphea exerts a negative influence on HRQOL, particularly in the domain of emotions and that patients have significant concern about the systemic implications of morphea. With this in mind, providers can be better equipped to recognize and address the components of morphea most worrisome to their patients.

\section{Acknowledgments}

We are indebted to Rose Cannon, Daniel Grabell, Simer Grewal, Andrew Kim, Kara Pretzlaff, and Rebecca Vasquez for their invaluable contributions.

Funding Sources:

Research for this manuscript was supported in part by NIH Grant No. K23AR056303-4. 


\section{References}

1. Finlay AY. Quality of life assessments in dermatology. Seminars in Cutaneous Medicine and Surgery. 1998; 17:291-6. [PubMed: 9859917]

2. Grozdev I, Kast D, Cao L, et al. Physical and mental impact of psoriasis severity as measured by the compact Short Form-12 Health Survey (SF-12) quality of life tool. The Journal of investigative dermatology. 2012; 132:1111-6. [PubMed: 22205305]

3. Klein R, Moghadam-Kia S, Taylor L, et al. Quality of life in cutaneous lupus erythematosus. J Am Acad Dermatol. 2011; 64:849-58. [PubMed: 21397983]

4. Deshpande PR, Rajan S, Sudeepthi BL, et al. Patient-reported outcomes: A new era in clinical research. Perspectives in clinical research. 2011; 2:137-44. [PubMed: 22145124]

5. Orzechowski NM, Davis DM, Mason TG 3rd, et al. Health-related quality of life in children and adolescents with juvenile localized scleroderma. Rheumatology (Oxford, England). 2009; 48:670-2.

6. Saxton-Daniels S, Jacobe HT. An evaluation of long-term outcomes in adults with pediatric-onset morphea. Archives of dermatology. 2010; 146:1044-5. [PubMed: 20855712]

7. Uziel Y, Laxer RM, Krafchik BR, et al. Children with morphea have normal self-perception. The Journal of pediatrics. 2000; 137:727-30. [PubMed: 11060543]

8. Das S, Bernstein I, Jacobe H. Correlates of self-reported quality of life in adults and children with morphea. J Am Acad Dermatol. 2014; 70:904-10. [PubMed: 24534655]

9. Kroft EB, de Jong EM, Evers AW. Psychological distress in patients with morphea and eosinophilic fasciitis. Archives of dermatology. 2009; 145:1017-22. [PubMed: 19770441]

10. Kroft EB, de Jong EM, Evers AW. Physical burden of symptoms in patients with localized scleroderma and eosinophilic fasciitis. Archives of dermatology. 2008; 144:1394-5. [PubMed: 18936410]

11. Szramka-Pawlak B, Danczak-Pazdrowska A, Rzepa T, et al. Health-related quality of life, optimism, and coping strategies in persons suffering from localized scleroderma. Psychology, health \& medicine. 2013

12. Johnson W, Jacobe H. Morphea in adults and children cohort II: patients with morphea experience delay in diagnosis and large variation in treatment. J Am Acad Dermatol. 2012; 67:881-9. [PubMed: 22382198]

13. Furst DE, Clements PJ, Steen VD, et al. The modified Rodnan skin score is an accurate reflection of skin biopsy thickness in systemic sclerosis. The Journal of rheumatology. 1998; 25:84-8. [PubMed: 9458208]

14. Arkachaisri T, Vilaiyuk S, Torok KS, et al. Development and initial validation of the localized scleroderma skin damage index and physician global assessment of disease damage: a proof-ofconcept study. Rheumatology (Oxford, England). 2010; 49:373-81.

15. Laxer RM, Zulian F. Localized scleroderma. Current opinion in rheumatology. 2006; 18:606-13. [PubMed: 17053506]

16. Chren MM, Lasek RJ, Flocke SA, et al. Improved discriminative and evaluative capability of a refined version of Skindex, a quality-of-life instrument for patients with skin diseases. Archives of dermatology. 1997; 133:1433-40. [PubMed: 9371029]

17. Basra MK, Fenech R, Gatt RM, et al. The Dermatology Life Quality Index 1994-2007: a comprehensive review of validation data and clinical results. The British journal of dermatology. 2008; 159:997-1035. [PubMed: 18795920]

18. Ware JE Jr, Sherbourne CD. The MOS 36-item short-form health survey (SF-36). I. Conceptual framework and item selection. Medical care. 1992; 30:473-83. [PubMed: 1593914]

19. Katz JN, Chang LC, Sangha O, et al. Can comorbidity be measured by questionnaire rather than medical record review? Medical care. 1996; 34:73-84. [PubMed: 8551813]

20. Sangha O, Stucki G, Liang MH, et al. The Self-Administered Comorbidity Questionnaire: a new method to assess comorbidity for clinical and health services research. Arthritis and rheumatism. 2003; 49:156-63. [PubMed: 12687505] 
21. Evers AW, Duller P, van de Kerkhof PC, et al. The Impact of Chronic Skin Disease on Daily Life (ISDL): a generic and dermatology-specific health instrument. Br J Dermatol. 2008; 158:101-8. [PubMed: 17999699]

22. Goreshi R, Chock M, Foering K, et al. Quality of life in dermatomyositis. J Am Acad Dermatol. 2011; 65:1107-16. [PubMed: 21722989]

23. Gniadecki R, Robertson D, Molta CT, et al. Self-reported health outcomes in patients with psoriasis and psoriatic arthritis randomized to two etanercept regimens. J Eur Acad Dermatol Venereol. 2012; 26:1436-43. [PubMed: 22035157]

24. Arkachaisri T, Vilaiyuk S, Li S, et al. The localized scleroderma skin severity index and physician global assessment of disease activity: a work in progress toward development of localized scleroderma outcome measures. The Journal of rheumatology. 2009; 36:2819-29. [PubMed: 19833758]

25. American Fact Finder. U.S. Census Bureau.

26. Jolly M, Mikolaitis RA, Shakoor N, et al. Education, zip code-based annualized household income, and health outcomes in patients with systemic lupus erythematosus. The Journal of rheumatology. 2010; 37:1150-7. [PubMed: 20360192]

27. Harnick DJ, Cohen JL, Schechter CB, et al. Effects of Practice Setting on Quality of LipidLowering Management in Patients With Coronary Artery Disease. The American Journal of Cardiology. 1998; 81:1416-20. [PubMed: 9645890]

28. Heffernan DS, Vera RM, Monaghan SF, et al. Impact of socioethnic factors on outcomes following traumatic brain injury. The Journal of trauma. 2011; 70:527-34. [PubMed: 21610339]

29. Klein R, Moghadam-Kia S, Taylor L, et al. Quality of life in cutaneous lupus erythematosus. Journal of the American Academy of Dermatology. 2011; 64:849-58. [PubMed: 21397983]

30. Ware, JE.; Snow, KK.; Kosinski, M., et al. SF-36 health survey: manual and interpretation guide. The Health Institute, New England Medical Center; 1993.

31. Hudson M, Thombs BD, Steele R, et al. Quality of life in patients with systemic sclerosis compared to the general population and patients with other chronic conditions. The Journal of rheumatology. 2009; 36:768-72. [PubMed: 19228662]

32. Ware, JEKM.; Keller, SK. SF-36® Physical and Mental Health Summary Scales: A User's Manual. Boston, MA: 1994.

33. Wolfe F, Michaud K, Li T, et al. EQ-5D and SF-36 quality of life measures in systemic lupus erythematosus: comparisons with rheumatoid arthritis, noninflammatory rheumatic disorders, and fibromyalgia. The Journal of rheumatology. 2010; 37:296-304. [PubMed: 20032098]

34. Becker N, Bondegaard Thomsen A, Olsen AK, et al. Pain epidemiology and health related quality of life in chronic non-malignant pain patients referred to a Danish multidisciplinary pain center. Pain. 1997; 73:393-400. [PubMed: 9469530]

35. Zachariae R, Lei U, Haedersdal M, et al. Itch severity and quality of life in patients with pruritus: preliminary validity of a Danish adaptation of the itch severity scale. Acta dermato-venereologica. 2012; 92:508-14. [PubMed: 22002738]

36. Kini SP, DeLong LK, Veledar E, et al. The impact of pruritus on quality of life: The skin equivalent of pain. Archives of dermatology. 2011; 147:1153-6. [PubMed: 21680760]

37. Xuan J, Kirchdoerfer LJ, Boyer JG, et al. Effects of comorbidity on health-related quality-of-life scores: an analysis of clinical trial data. Clinical therapeutics. 1999; 21:383-403. [PubMed: 10211540]

38. Burstrom K, Johannesson M, Diderichsen F. Health-related quality of life by disease and socioeconomic group in the general population in Sweden. Health policy (Amsterdam, Netherlands). 2001; 55:51-69.

39. Haider A, Mamdani M, Shaw JC, et al. Socioeconomic status influences care of patients with acne in Ontario, Canada. J Am Acad Dermatol. 2006; 54:331-5. [PubMed: 16443069] 


\section{What's already known about this topic?}

- Little is known about HRQOL in morphea.

- Existing studies indicate morphea has modest effect on life quality in children, while studies in adults generally report greater negative impact on life quality and emotional distress.

\section{What does this study add?}

- Disease severity and symptoms such as pain and itch are linked with HRQOL. Pruritus reflects disease activity. Individuals with morphea worry about the impact and progression of their condition.

- HRQOL issues in morphea should be addressed. Physicians have the opportunity to intervene with education about the disease and its prognosis. 
Skinde $x-29+3$
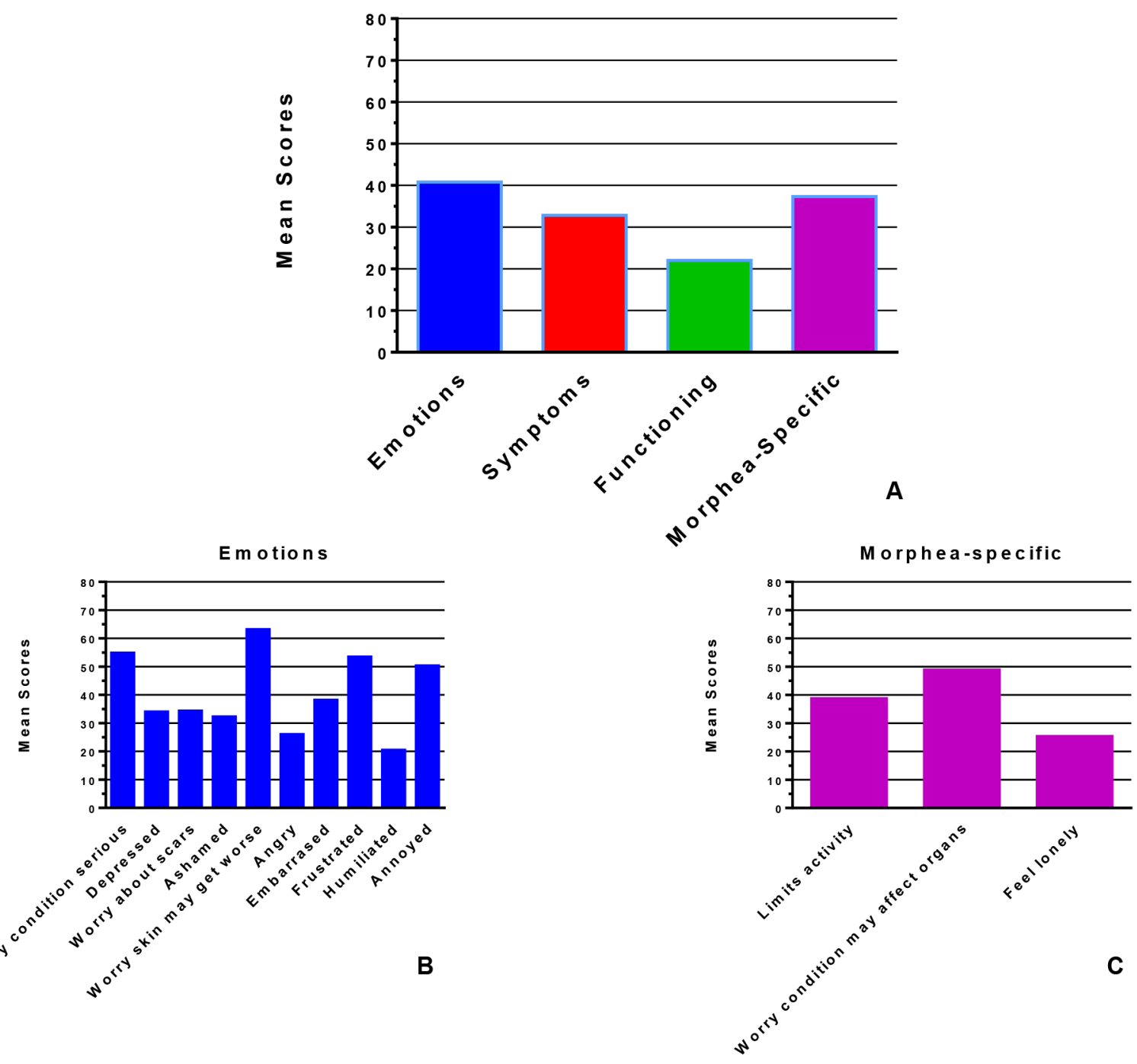

Figure 1.

Details of HRQOL in morphea as assessed by the Skindex-29+3. Mean skindex subscale scores (A) and mean scores of individual questions within the (B) emotions, and (C) morphea-specific subscales are depicted. 
E th n ic ity

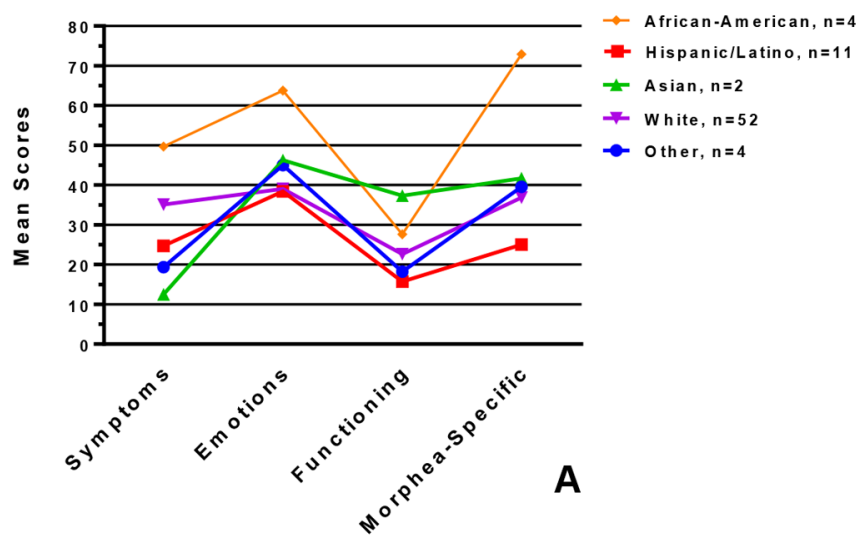

Insurance Type

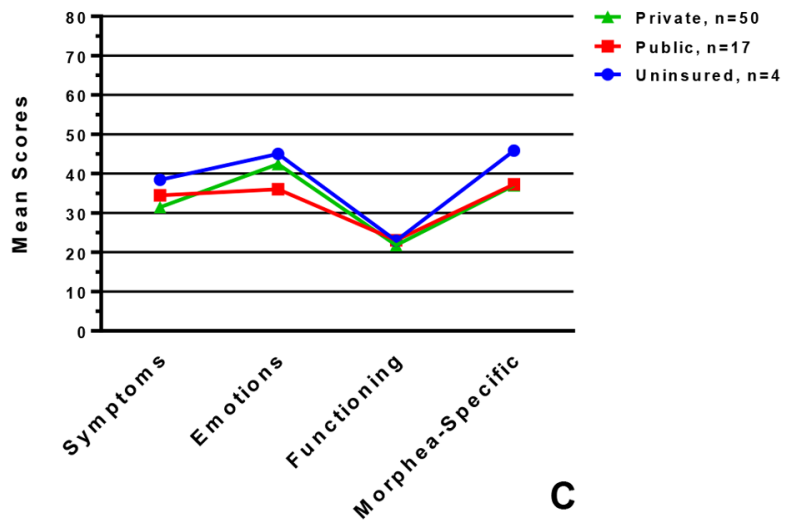

Subtype

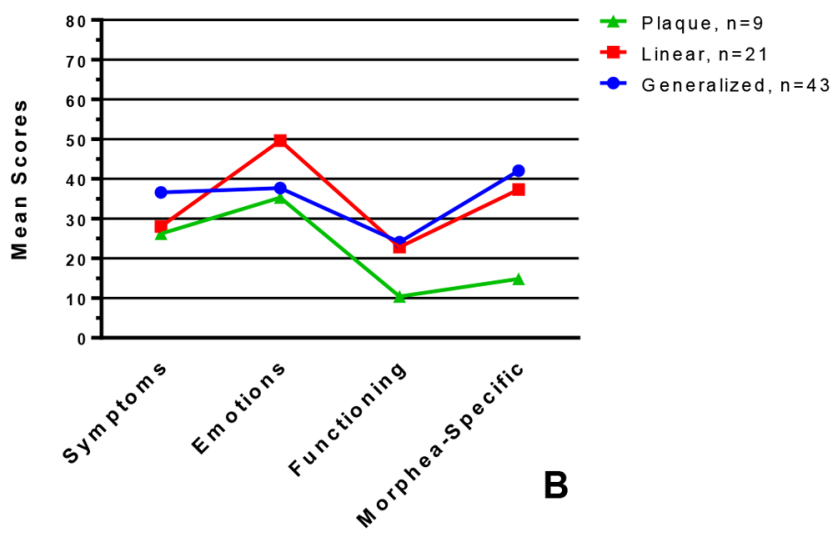

Household Income

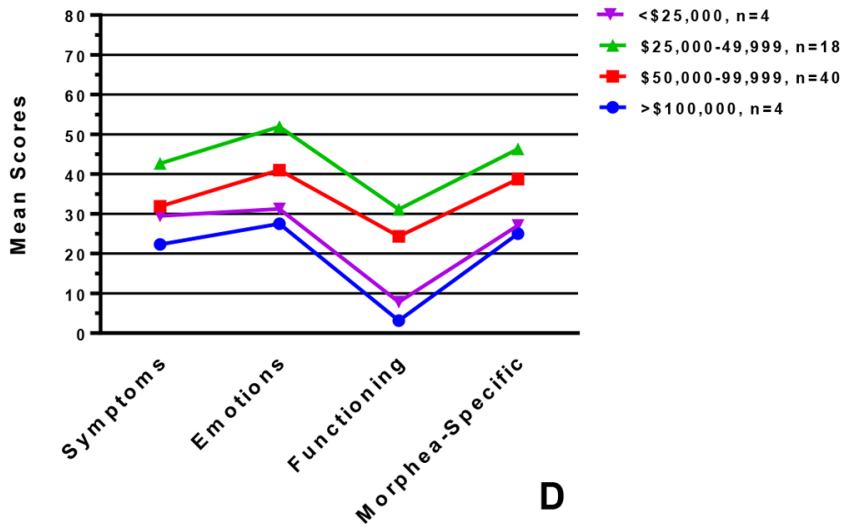

Figure 2.

Factors related to HRQOL as measured by the Skindex-29+3. Mean Skindex-29+3 scores are given for (A) ethnicity, (B) Z-AHI, (C) insurance type (unavailable for 2 patients) and (D) disease severity. Z-AHI, Zip-code based annualized household income (unavailable for 7 patients). 


\section{Table 1}

Patient characteristics

\begin{tabular}{|c|c|c|}
\hline Age Mean Yrs, (SD) & $41.3(18.2)$ & \\
\hline \multicolumn{3}{|l|}{ Gender n, \% } \\
\hline Female & 62 & $85 \%$ \\
\hline Male & 11 & $15 \%$ \\
\hline \multicolumn{3}{|l|}{ Ethnicity n, \% } \\
\hline White & 52 & $71 \%$ \\
\hline Hispanic/Latino & 11 & $15 \%$ \\
\hline African American & 4 & $5 \%$ \\
\hline Asian and Others & 6 & $8 \%$ \\
\hline \multicolumn{3}{|l|}{ Income n, \% } \\
\hline Less than $\$ 25,000$ & 4 & $6 \%$ \\
\hline$\$ 25,000-49,999$ & 18 & $27 \%$ \\
\hline$\$ 50,000-100,000$ & 40 & $61 \%$ \\
\hline Over $\$ 100,000$ & 4 & $6 \%$ \\
\hline \multicolumn{3}{|l|}{ Insurance Status n, \% } \\
\hline No Insurance & 4 & $6 \%$ \\
\hline Public & 17 & $24 \%$ \\
\hline Private & 50 & $70 \%$ \\
\hline \multicolumn{3}{|l|}{ Morphea Subtype n, \% } \\
\hline Plaque & 9 & $12 \%$ \\
\hline Linear & 21 & $29 \%$ \\
\hline Generalized & 43 & $59 \%$ \\
\hline \multicolumn{3}{|l|}{ LoSCAT mean (SD) } \\
\hline LoSSI & 12 & (15) \\
\hline PGA-A & 28 & (27) \\
\hline LoSDI (mean) & 19 & (16) \\
\hline PGA-D & 30 & (22) \\
\hline MRSS & 6 & (5) \\
\hline
\end{tabular}

Current Therapy n, \%

\begin{tabular}{lll}
\hline Topical/Intralesional (IL) Therapy & 19 & $26 \%$ \\
Systemic Agents/Phototherapy & 16 & $22 \%$ \\
Combination Treatment & 14 & $19 \%$ \\
No Current Therapy & 24 & $33 \%$ \\
\hline
\end{tabular}

Br J Dermatol. Author manuscript; available in PMC 2016 May 01. 
Nine patients were excluded from LoSSI analysis, 7 from PGA-A analysis, 6 from MRSS analysis, 9 from LoSDI analysis, and 8 from PGA-D analysis as these scores were not calculated on same visit as HRQOL instrument administration. Income and insurance data were unavailable for 7 and 2 patients, respectively.

LoSCAT, localized scleroderma cutaneous assessment tool; LoSDI, Localized Scleroderma Skin Damage Index; LoSSI, Localized Scleroderma Skin Severity Index; MRSS, modified Rodnan skin score; PGA-A, Physician Global Assessment-Activity; PGA-D, Physician Global AssessmentDamage; $I L$, intralesional. 


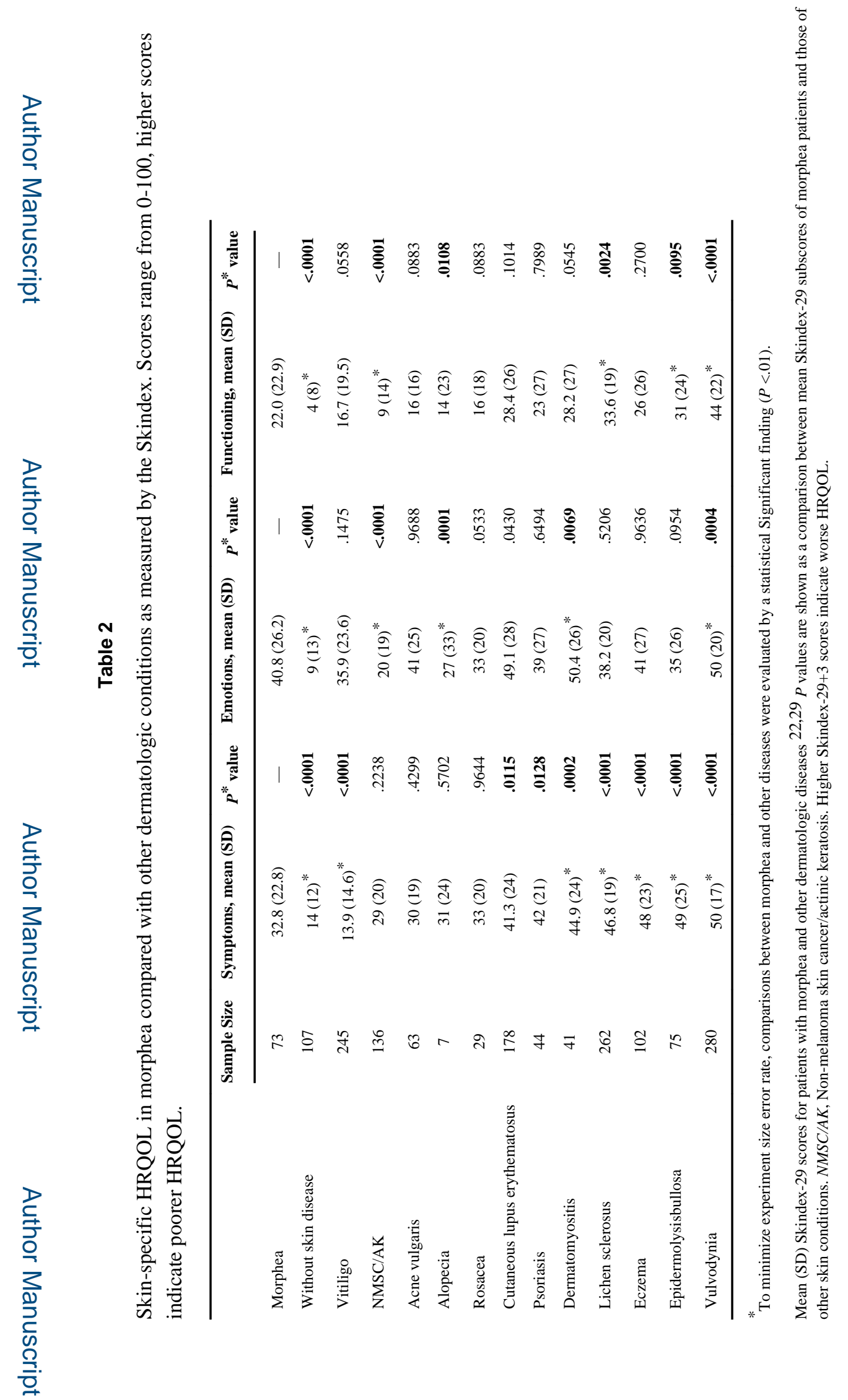

Br J Dermatol. Author manuscript; available in PMC 2016 May 01. 


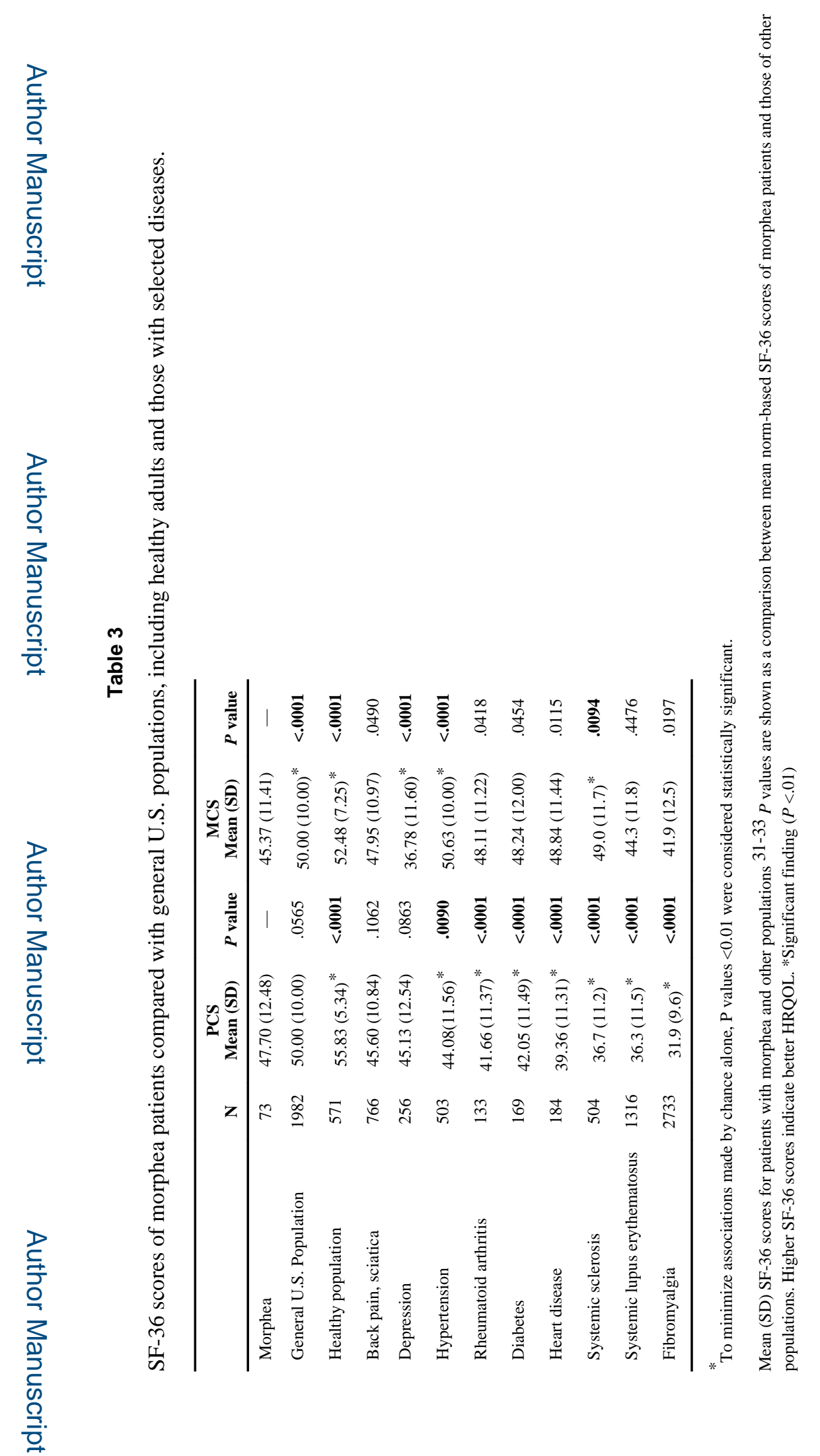

Br J Dermatol. Author manuscript; available in PMC 2016 May 01. 


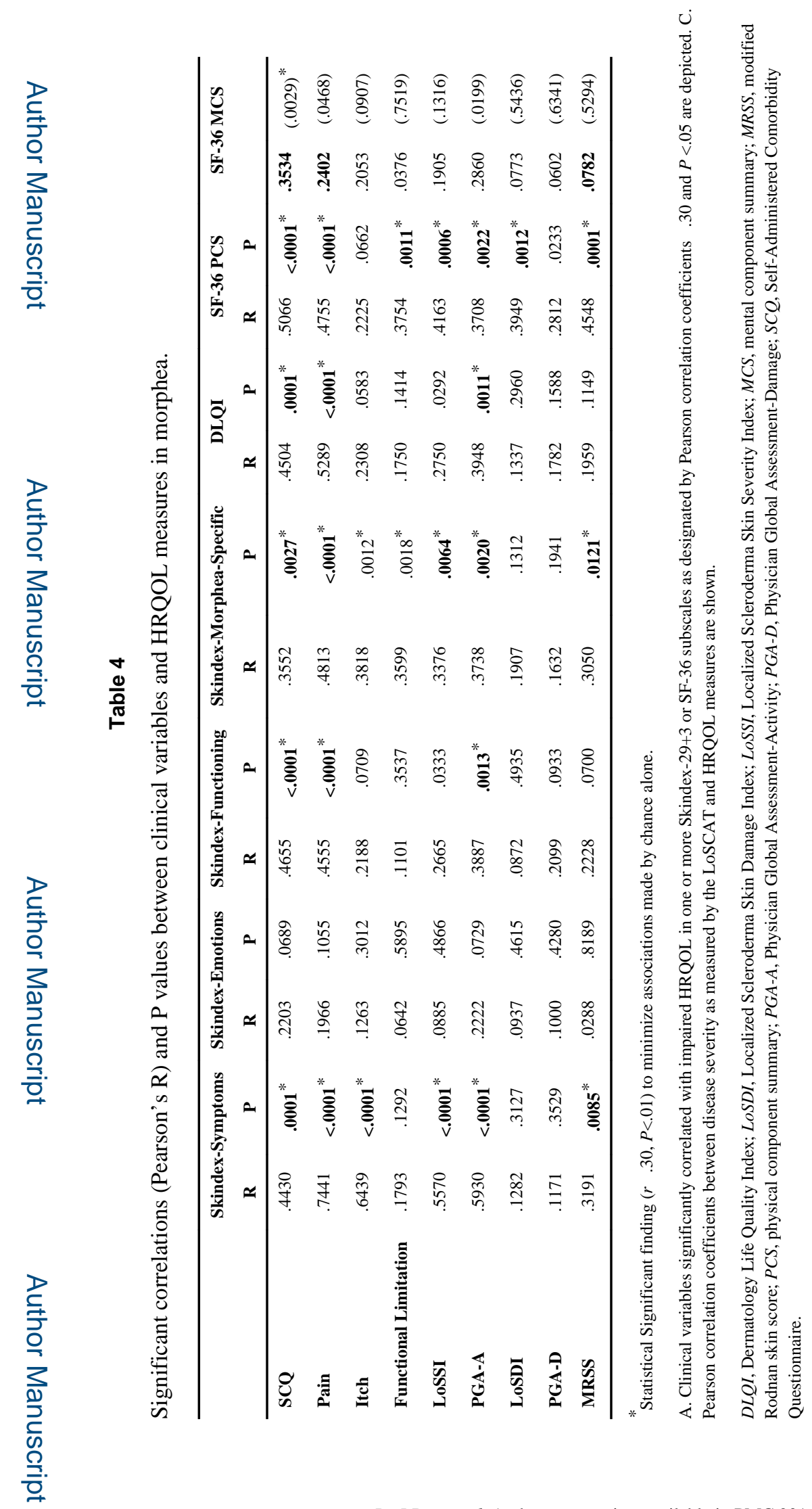

Br J Dermatol. Author manuscript; available in PMC 2016 May 01. 